\title{
Meios de proteção contra radiação utilizados em estabelecimentos de assistência à saúde odontológica
}

\section{Radiation protection means used in dental health care establishments}

\author{
Dayse Hanna Maia Oliveira* \\ Manuella Santos Carneiro Almeida* \\ Camila Helena Machado da Costa ${ }^{* * *}$ \\ Luis Ferreira de Sousa Filho ${ }^{* * *}$
}

\section{Resumo}

Objetivo: avaliar os procedimentos dos cirurgiões-dentistas acerca dos aspectos de radioproteção na cidade de Patos, PB, de acordo com a legislação sanitária brasileira. Materiais e método: foi utilizada uma abordagem indutiva com procedimento descritivo e técnica de pesquisa por documentação direta em campo. Na cidade de Patos, entre os 43 consultórios odontológicos existentes, 35 proprietários aceitaram participar da pesquisa e assinaram o termo de consentimento livre e esclarecido. O pesquisador, após autorização, realizou inspeção visual e preencheu o formulário contendo itens concernentes à radioproteção. Resultados: foi constatada a ausência de sinalização da radiação ionizante nas salas de raios- $X$ em 94,2\% dos consultórios e em nenhum estabelecimento foi identificado o aviso acerca da importância da ciência do dentista em casos de pacientes gestantes. Foi observado, também, aventais de chumbo acondicionados de maneira incorreta e até mesmo consultórios que não dispunham de aventais e protetores de tireoide. Conclusão: alguns aspectos de radioproteção estão em desacordo com a legislação sanitária brasileira nos consultórios odontológicos de Patos, demonstrando que existem cirurgiões-dentistas que desconhecem ou não cumprem algumas normas de radioproteção vigentes, que poderiam minimizar os riscos inerentes da exposição à radiação.

Palavras-chave: Proteção radiológica. Radiologia. Vigilância Sanitária.

\section{Introdução}

Desde a descoberta dos raios $\mathrm{X}$ por Wilhelm Conrad Röntgen, em novembro de 1895, várias pesquisas enaltecem a importância dessa radiação para a humanidade, bem como suas ações deletérias devido à ação ionizante ${ }^{1}$. A comunidade científica, após observar a existência de tais danos, passou a estudar a proteção radiológica, regulamentando e limitando o uso das radiações em condições aceitáveis com a finalidade de proteger os indivíduos ${ }^{2}$.

A prevenção em qualquer procedimento envolvendo a radiação ionizante torna-se fundamental visto que ela não é percebida pelos sentidos humanos e qualquer que seja o nível de exposição pode provocar algum tipo de dano biológico ao paciente ${ }^{3}$. Os efeitos da radiação dependem da quantidade e da qualidade da radiação incidente e, também, da natureza do material com a qual está interagindo ${ }^{4}$.

Muitos países têm leis elaboradas para o controle da prática odontológica em relação aos raios X. No Brasil, apenas em 1998, a Portaria no 453 do Ministério da Saúde estabeleceu um conjunto de diretrizes para que os serviços de radiologia trabalhassem com segurança e qualidade ${ }^{5}$. Essa norma preconiza a eficácia nos procedimentos de radiodiagnóstico prestados à população, assim como as-

\footnotetext{
Aluna de graduação do Departamento de Odontologia, Universidade Federal de Campina Grande, Patos, Paraíba, Brasil.

Professora doutora do Departamento de Odontologia, Universidade Federal de Campina Grande, Patos, Paraíba, Brasil.

*** Professora doutora do Departamento de Odontologia da Universidade Federal de Campina Grande, Patos, Paraíba, Brasil.

**** Médico clínico geral do Hospital Regional de Patos, Patos, Paraíba, Brasil.
} 
segura os requisitos mínimos de proteção radiológica aos pacientes e profissionais da área de saúde para que trabalhem sem riscos e com eficiência ${ }^{1}$.

A portaria exige o atendimento de uma série de itens relacionados à precisão e à constância dos equipamentos geradores dos raios $\mathrm{X}$ e com os procedimentos de trabalho. As leis de proteção radiológica seguem o princípio ALARA (As Low As Reasonnably Achievable - tão baixo quanto razoavelmente possível), defendendo a utilização de medidas que minimizem a exposição à radiação $0^{5}$.

O exame radiográfico é uma importante ferramenta complementar utilizada na investigação diagnóstica na área da saúde ${ }^{6}$. A radiografia periapical é bastante utilizada nas diversas especialidades odontológicas em manobras clínicas pré, trans ou pós-operatórias, além de auxiliar no diagnóstico precoce de patologias da cavidade oral, de maneira a ser o responsável por $20 \%$ do número total de exames radiográficos realizados no país ${ }^{1,7}$.

Alguns estudos têm sido desenvolvidos com o intuito de avaliar a adoção de medidas de radioproteção, sendo de grande relevância para a saúde pública ${ }^{1,6,8,9-11}$. É importante assinalar a existência de estudos regionais que busquem avaliar os cuidados adotados para minimizar a exposição aos raios $\mathrm{X}$ e a maneira como essa radiação está sendo utilizada nos consultórios, observando se o cirurgião-dentista conhece e segue as normas estabelecidas pela lei nacional.

Diante disso, foi objetivo do presente trabalho avaliar os procedimentos dos cirurgiões-dentistas acerca dos aspectos de radioproteção na cidade de Patos, PB, de acordo com a legislação sanitária brasileira e também identificar os aspectos mais deficientes nos consultórios odontológicos.

\section{Materiais e método}

Foi utilizada uma abordagem indutiva com procedimento descritivo e técnica de pesquisa por documentação direta em campo. Foram incluídos na pesquisa todos os consultórios odontológicos que dispunham de aparelhos de raios $\mathrm{X}$ intraoral da cidade de Patos, na Paraíba. Tratou-se de uma pesquisa transversal e quantitativa que foi realizada no período de outubro de 2013 a abril de 2015, após aprovação do Comitê de Ética em Pesquisa via Plataforma Brasil.

Foram inspecionados neste estudo os consultórios odontológicos que obedeceram aos seguintes critérios:

- Dispor de aparelho de raios $\mathrm{X}$ intraoral em condições de funcionamento;

- Realizar ordinariamente exposições radiográficas na prática odontológica;

- Possuir um profissional com curso superior em Odontologia como responsável técnico pelo consultório;

- Estar situado na cidade de Patos, PB.
Dos 43 consultórios odontológicos existentes na cidade, 35 proprietários aceitaram participar da pesquisa e assinaram o termo de consentimento livre e esclarecido. Foi preenchido pelo pesquisador mediante inspeção visual, o formulário concernente às atitudes dos cirurgiões-dentistas quanto à radioproteção, que compreendia os seguintes itens: 1) Existência de sinalização de radiações ionizantes (trifólio) na sala; 2) Existência de aviso aos pacientes e acompanhantes a respeito da necessidade de utilização do avental plumbífero; 3) Existência de aviso acerca da importância da ciência do cirurgião-dentista em casos de pacientes gestantes; 4) Existência de avental plumbífero; 5) Existência do protetor de tireoide; 6) Equivalência de chumbo das vestimentas plumbíferas; 7) Forma de acondicionamento do avental de chumbo. Durante a visita, o profissional foi questionado quanto à medida de radioproteção que adotava durante as exposições radiográficas.

Ao final da inspeção, os profissionais foram orientados verbalmente quanto às melhorias que poderiam ser realizadas referentes aos aspectos de radioproteção. Os dados obtidos no presente estudo foram tabulados, utilizando o programa Microsoft Excel $2013^{\circledR}$, analisados de forma descritiva e resumidos por meio de frequências absolutas e relativas percentuais.

\section{Resultados}

Após a análise dos dados, foram observados os resultados apresentados nas tabelas a seguir.

Tabela 1 - Avisos para pacientes e acompanhantes sobre a proteção contra radiação nos consultórios odontológicos de Patos, PB, Brasil, 2013-2015

\begin{tabular}{l|l}
\hline \multicolumn{1}{c|}{ Questões } & \multicolumn{1}{c}{ Respostas } \\
\hline $\begin{array}{l}\text { Existe sinalização de radiações ionizantes } \\
\text { (trifólio) na porta da sala? }\end{array}$ & $\begin{array}{l}\text { Sim }-5,8 \% \\
\text { Não }-94,2 \%\end{array}$ \\
\hline $\begin{array}{l}\text { Existe aviso aos pacientes e acompanhantes } \\
\text { acerca da necessidade de utilização do } \\
\text { avental plumbífero? }\end{array}$ & Sim $-14,3 \%$ \\
Não $-85,7 \%$ \\
\hline $\begin{array}{l}\text { Existe aviso acerca da importância da } \\
\text { ciência do dentista em casos de pacientes } \\
\text { gestantes? }\end{array}$ & Sim $-0 \%$ \\
\hline
\end{tabular}

Fonte: elaboração dos autores.

Tabela 2 - Meios de proteção contra radiação utilizados por pacientes nos consultórios odontológicos de Patos, PB, Brasil 2013-2015

\begin{tabular}{l|l}
\hline \multicolumn{1}{c|}{ Questões } & \multicolumn{1}{c}{ Respostas } \\
\hline Existe avental plumbífero no consultório? & $\begin{array}{l}\text { Sim }-97,1 \% \\
\text { Não }-2,9 \%\end{array}$ \\
\hline $\begin{array}{l}\text { Existe avental protetor de tireoide no } \\
\text { consultório? }\end{array}$ & $\begin{array}{l}\text { Sim }-88,5 \% \\
\text { Não }-11,5 \%\end{array}$ \\
\hline $\begin{array}{l}\text { As vestimentas plumbíferas possuem } \\
\text { equivalência de chumbo adequada } \\
\text { (mínimo de } 0,25 \mathrm{mmPb} \text { )? }\end{array}$ & $\begin{array}{l}\text { Sim }-100 \% \\
\text { Não }-0 \%\end{array}$ \\
\hline
\end{tabular}

Fonte: elaboração dos autores. 
Tabela 3 - Formas de acondicionamento do avental de chumbo nos consultórios odontológicos de Patos, PB, Brasil, 20132015

\begin{tabular}{l|l}
\hline \multicolumn{1}{c|}{ Questão } & \multicolumn{1}{c}{ Respostas } \\
\hline & Estendido $-20,0 \%$ \\
De que forma o avental & Dobrado $-71,4 \%$ \\
plumbífero é acondionado? & No chão $-5,7 \%$ \\
& Não possui $-2,9 \%$ \\
\hline
\end{tabular}

Fonte: elaboração dos autores.

Tabela 4 - Meios de proteção contra radiação utilizados pelos cirurgiões-dentistas dos consultórios odontológicos de Patos, PB, Brasil, 2013-2015

\begin{tabular}{l|l}
\hline \multicolumn{1}{c|}{ Questão } & \multicolumn{1}{c}{ Respostas } \\
\hline & Afasta-se $-34,2 \%$ \\
Quais medidas de & Permanece atrás da parede $-65,7 \%$ \\
proteção o profis- & Utiliza avental de chumbo $-5,7 \%$ \\
sional realiza du- & Nenhuma $-0 \%$ \\
rante a exposição & $*$ Alguns profissionais utilizavam \\
radiográfica? & mais de uma forma de proteção \\
\hline
\end{tabular}

Fonte: elaboração dos autores.

\section{Discussão}

Apesar da Portaria no 453/1998 estar em vigor há dezessete anos, não são encontrados muitos levantamentos na literatura sobre radioproteção no Brasil $^{5}$. Tendo em vista a importância do assunto para a saúde dos pacientes e dos profissionais envolvidos na execução de exames radiográficos, torna-se necessária a realização de trabalhos para verificar a adequação dos consultórios das cidades brasileiras ao que estabelece a legislação. A partir dos resultados obtidos nesta pesquisa, foi possível verificar algumas irregularidades nos consultórios odontológicos da cidade de Patos, Paraíba.

De acordo com o que é determinado pela lei, os profissionais da saúde que fazem uso de qualquer tipo de radiação ionizante, seja para diagnóstico ou terapia, têm o dever de proteger seus pacientes com a blindagem plumbífera para evitar a exposição das gônadas, tórax e tireoide ${ }^{8}$. No presente estudo, foi possível identificar que, na maioria dos consultórios vistoriados $(97,1 \%)$, os cirurgiões-dentistas faziam uso da vestimenta plumbífera para proteção dos pacientes. Resultado semelhante foi observado no estudo de Castro et al. ${ }^{9}$ (2013), em Belo Horizonte, no qual a medida de radioproteção referente ao uso do avental de chumbo era respeitada por $96,4 \%$ dos dentistas. Já Neves et al. ${ }^{5}$ (2010) observaram que apenas $79 \%$ dos consultórios odontológicos averiguados de São José do Rio Preto, São Paulo, faziam uso do equipamento.

Apesar da recomendação de utilização obrigatória da proteção plumbífera ${ }^{8}$, ainda foi possível constatar que em 2,9\% dos consultórios não havia o item. No estudo de Castro et al. ${ }^{9}$ (2013), o per- centual era de 3,6\%. Esse aspecto é frequentemente alvo de notificações e interdições sanitárias, uma vez que contraria os princípios de radioproteção que a Portaria no $453 / 1998^{5}$ preconiza.

A forma inadequada de guardar o protetor de chumbo pode danificá-lo, devido à formação de fendas no material, o que pode comprometer sua função, que passará a não acontecer de maneira adequada $^{5}$. Foi possível observar que na maior parte dos consultórios avaliados $(74,3 \%)$ o avental plumbífero não era acondicionado de maneira correta, já que foi encontrado dobrado em suportes fixos na parede, apoiados sobre o braço do aparelho de raios $\mathrm{X}$ e, até mesmo, no chão. Resultado semelhante foi encontrado na pesquisa de Flôr e Kirchhof ${ }^{4}$ (2006), que registrou, além da escassez de equipamentos plumbíferos de proteção individual, a conservação inadequada do equipamento, que foi encontrado, em muitos consultórios, dobrado e depositado sobre móveis. Já o estudo de Castro et al. ${ }^{9}$ (2013) contrapõe-se a esses resultados, pois a maioria dos dentistas $(56,5 \%)$ armazenava adequadamente o avental de chumbo.

A lei recomenda o uso obrigatório para o paciente do protetor plumbífero específico para a área da tireoide $^{8}$, entretanto, foi observado que alguns dos consultórios (4) não dispunham o equipamento. Em estudo realizado em 1997, Hopper afirmou que um protetor de tireoide consegue atenuar até $67,3 \%$ da radiação $\mathrm{X}^{11}$, o que o torna importante na proteção do paciente, considerando a série de efeitos deletérios que os raios $\mathrm{X}$ podem provocar nos seres vivos, independentemente da quantidade de exposição $0^{3,4}$. $\mathrm{O}$ item 4.26a (ii) da Portaria $\mathrm{n}^{\circ} 453$ recomenda que as vestimentas plumbíferas, para serem capazes de atenuar a radiação ionizante, devem possuir no mínimo $0,25 \mathrm{mmPb}^{11}$. Todas as barreiras plumbíferas do presente estudo apresentaram equivalência de chumbo adequada, assim como determina a Portaria $\mathrm{n}^{\mathrm{o}} 453^{11}$.

Para proteção própria, observou-se que grande parte dos cirurgiões-dentistas se distanciava da fonte de radiação durante a realização do exame radiográfico e que a maioria preferia ficar atrás de alguma parede fora da sala de exame. É indicado que, ao realizar exames intrabucais, o operador mantenha-se a uma distância de, pelo menos, dois metros do tubo e do paciente durante as tomadas, uma vez que o poder de penetração das radiações ionizantes é inversamente proporcional ao quadrado da distância ${ }^{1,4}$.

Ainda com relação à proteção do profissional,

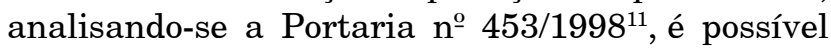
entender que não há necessidade do uso de dosímetro por esses profissionais e que o dispositivo é desnecessário em consultórios que realizam poucas radiografias semanais (aparelho emitindo menos do que $4 \mathrm{~mA} / \mathrm{min} /$ semana). O equipamento de proteção é necessário apenas para o uso em aparelhos que emitem maior quantidade de radiação, como apare- 
lhos extrabucais e tomógrafos. No presente estudo, nenhum profissional utilizou o filme dosimétrico.

Além da distância da fonte de radiação, também é recomendado o uso do avental plumbífero pelo profissional. Apesar de terem sido identificados cirurgiões-dentistas que faziam uso do avental de chumbo, foi possível verificar desconhecimento, e até certa resistência dos profissionais quanto ao uso da vestimenta devido ao desconforto provocado pelo peso, que pode ocasionar dores lombares quando utilizado por longos períodos ${ }^{11}$.

Para proteção do público em geral, o Ministério da Saúde indica que as salas equipadas com aparelhos de raios $\mathrm{X}$ devem dispor de sinalização visível no exterior das portas de acesso, contendo o símbolo internacional da radiação ionizante (trifólio) acompanhado da inscrição "Raios X, entrada restrita" ou "Raios X, entrada proibida a pessoas não autorizadas" e um quadro com orientações sobre o uso de vestimenta plumbífera ${ }^{1}$. Apesar dessa determinação, na maioria dos consultórios visitados, foi verificada a ausência do trifólio e, em grande parte, também não foi encontrada qualquer recomendação aos pacientes a respeito da necessidade de utilização do avental de chumbo para pacientes ou acompanhantes. A ausência de sinalização ionizante nas salas de raios $\mathrm{X}$ é uma negligência considerada grave, pois as pessoas que estão nos arredores do local onde ocorrem as tomadas podem ser expostas, por acidente, por desconhecerem que aquela área é restrita e oferece riscos ${ }^{1}$.

Sabe-se, ainda, que a exposição aos raios $\mathrm{X}$ é extremamente danosa ao feto, podendo provocar até morte uterina prematura, quando ocorre no primeiros dias pós-concepção, também, durante o primeiro trimestre, as radiografias devem ser evitadas devido à velocidade de replicação celular ${ }^{12}$. Os avisos sobre a realização de exames radiográficos em pacientes gestantes têm o objetivo de informá-las sobre a segurança desse procedimento, no caso de a avaliação do cirurgião-dentista ser de que tal exame é extremamente necessário. Quando os benefícios sobrepujarem os malefícios, o exame poderá ser realizado, desde que se obedeça a determinadas restrições, como evitar repetições e proteger o abdome da paciente com avental de chumbo ${ }^{12}$. Apesar da importância desse tipo de aviso, no presente estudo, não foi encontrado em nenhum consultório visitado, o aviso acerca da importância da ciência do dentista em casos de pacientes gestantes.

Ademais, de forma predominante, os consultórios não exibiam avisos de forma geral. Esses resultados corroboram com os de Neves et al. ${ }^{5}$ (2010, que não encontraram o símbolo internacional da radiação ionizante e nem quadro de orientações sobre o uso de vestimenta plumbífera, casos desrespeitados, respectivamente, em $41 \%$ e $79 \%$ dos consultórios.

Dentre os resultados encontrados, destaca-se a existência de profissionais que ainda desconhecem normas básicas de radioproteção. Esses resultados corroboram com estudos realizados em Aracaju, SE, São José do Rio Preto, SP e Belo Horizonte-BH ${ }^{1,5,9}$. Muitas falhas que ocorrem na adoção das normas da portaria estão relacionadas à falta de conscientização dos profissionais quanto aos efeitos deletérios que a radiação $\mathrm{X}$ pode causar aos pacientes $\mathrm{e}$ à equipe profissional ${ }^{5}$. Portanto, é importante que o cirurgião-dentista conheça e siga as normas de proteção em seu consultório durante a execução de radiografias, na tentativa de minimizar a quantidade de radiação à qual o paciente será exposto, diminuindo, assim, os efeitos biológicos nocivos ao organismo ${ }^{5}$. Além disso, cabe aos profissionais conhecer esses efeitos biológicos dos raios $\mathrm{X}$ para minimizá-los a fim de usar a radiação como um meio seguro e auxiliar de diagnóstico ${ }^{13}$.

\section{Conclusão}

Foi observado que alguns aspectos de radioproteção estão em desacordo com a legislação sanitária brasileira nos consultórios odontológicos de Patos, $\mathrm{PB}$, demonstrando que existem cirurgiões-dentistas que desconhecem ou não cumprem algumas das normas de radioproteção vigentes. A aparente negligência de alguns profissionais sobre os aspectos de radioproteção constatada nos resultados desta pesquisa é considerada preocupante, de acordo com a legislação, pelos riscos oferecidos aos pacientes.

Os profissionais precisam se conscientizar quanto aos riscos potenciais da radiação $\mathrm{X}$ e aplicar corretamente os regulamentos de proteção radiológica, como rege a Portaria $n^{\circ} 453 / 1998$, pois quem a descumpre, além de expor a própria saúde e a dos pacientes, pode ser notificado, autuado e, até mesmo, ter seu consultório odontológico interditado pela autoridade sanitária.

\section{Abstract}

Objective: To assess the procedures of dental surgeons regarding radioprotection aspects in the city of Patos, $P B$, Brazil, according to the Brazilian health legislation. Materials and method: An inductive approach was used with a descriptive procedure and research technique by direct field documentation. Out of the 43 dental offices in the city of Patos, 35 agreed to participate in the research and signed the informed consent form. After authorization, the researcher conducted visual inspection and filled out the form containing items concerning radiation protection. Results: The absence of signs for ionizing radiation in X-ray rooms was found in $94.2 \%$ of the dental offices and none of the establishments presented a warning sign about the importance of dental science in cases of pregnant patients. Lead aprons were found incorrectly stowed, as well as dental offices with aprons and thyroid protectors. Conclusion: Some aspects of radiation protection do not comply with the Brazilian health legislation in dental offices of the city of 
Patos, PB, showing there are dentists who do not know or do not meet some of the current radiation protection standards, which could minimize inherent risks of radiation exposure.

Keywords: Radiation Protection. Radiology. Health Surveillance.

\section{Referências}

1. Melo MFB, Melo SLS. Condições de radioproteção dos consultórios odontológicos. Cien Saude Colet 2008; 13(2):2163-9.

2. Santos RA, Miranda AC, Silva EC. As normas de radioproteção e o uso dos equipamentos de proteção individual na concepção dos cirurgiões-dentistas. Cien Saude Colet 2010; 15(2):3125-7.

3. Okuno E. Efeitos biológicos das radiações ionizantes. Acidente radiológico de Goiânia. Estud Avançados 2013; 27(77):185-200.

4. Flôr RC, Kirchhof ALC. Uma prática educativa de sensibilização quanto à exposição à radiação ionizante com profissionais de saúde. Rev Bras Enferm 2006; 59(3):274-8.

5. Neves FS, Vasconcelos TV, Bastos LC, Góes LA, Freitas DQ. Atitudes dos cirurgiões-dentistas em relação à proteção radiológica, de acordo com a lei brasileira. Rev Odontol Brasileira Central 2010; 19(51):301-7.

6. Grigoletto JC, Santos CB, Albertini LB, Takayanagui AMM. Situação do gerenciamento de efluentes de processamento radiográfico em serviços de saúde. Radiol Bras 2011; 44(5):301-7.

7. Yacovenco A, Tavano O, Tauhata L, Yacovenco M. Análise crítica dos serviços que visam a implementação da Portaria 453 em radiologia odontológica. Revista ABRO 2004; 5(1):16-20.

8. Bohner LOL, Bohner TOL, Mafaldo IAC, Peres PEC, Rosa MB. Difusão de material informativo sobre o descarte de resíduos radiológicos entre acadêmicos de odontologia e cirurgiões-dentistas da região sul do Brasil. REMOA 2011; $3(3): 476-81$.

9. Castro MAA, Aguiar VLF, Santos CR, Abreu MV, Abdo EN, Ferreira EF. Avaliação da utilização de aparelhos de raios-x em consultórios odontológicos em Belo Horizonte, Brasil. Arq Odontol 2013; 49(4):191-7.

10. Gomes CK, Duque ACR, Dias IM, Martins MEMN, Devito KL. Avaliação do conhecimento dos usuários da Faculdade de Odontologia da UFJF quanto às medidas de radioproteção. Odontologia Clín-Cient 2012; 11(1):25-9.

11. Soares FAP, Pereira AG, Flôr RC. Utilização de vestimentas de proteção radiológica para redução de dose absorvida: uma revisão integrativa da literatura. Radiol Bras 2011; 44(2):97-103.

12. Caneppele TMF, Yamamoto EC, Souza AC, Valera MC, Araújo MAM. Conhecimento dos cirurgiões-dentistas sobre $o$ atendimento de pacientes especiais: hipertensos, diabéticos e gestantes. J Biodentist Biomat 2011; 1:31-1.

13. Oliveira GF, Neto MLC, Eid NLM, Pereira AC. Avaliação do conhecimento e dos procedimentos preventivos de radioproteção em consultórios odontológicos localizados na cidade de Sao Paulo. Revista ABRO 2005; 6(1):35-41.
Endereço para correspondência:

Manuella Santos Carneiro Almeida

Rua Ambrosina Soares dos Santos, 347, Bessa

58035-140 João Pessoa, PB

Telefone: (83) 99985-3233

E-mail: manuellacarneiro@hotmail.com

Recebido: 15/03/2016. Aceito: 08/08/2016. 